Research Article

\title{
Hermitian Positive Definite Solution of the Matrix Equation $X=Q+\sum_{i=1}^{m} A_{i}\left(B+X^{-1}\right)^{-1} A_{i}^{*}$
}

\author{
Chun-Mei Li ${ }^{1}$ and Jing-Jing Peng ${ }^{2}$ \\ ${ }^{1}$ College of Mathematics and Computational Science, Guilin University of Electronic Technology, Guilin 541004, China \\ ${ }^{2}$ College of Mathematics and Econometrics, Hunan University, Changsha 410082, China \\ Correspondence should be addressed to Chun-Mei Li; lengyue123@126.com
}

Received 26 May 2014; Revised 10 August 2014; Accepted 9 September 2014; Published 24 September 2014

Academic Editor: Asai Asaithambi

Copyright ( 2014 C.-M. Li and J.-J. Peng. This is an open access article distributed under the Creative Commons Attribution License, which permits unrestricted use, distribution, and reproduction in any medium, provided the original work is properly cited.

We consider the Hermitian positive definite solution of the nonlinear matrix equation $X=Q+\sum_{i=1}^{m} A_{i}\left(B+X^{-1}\right)^{-1} A_{i}^{*}$. Some new sufficient conditions and necessary conditions for the existence of Hermitian positive definite solutions are derived. An iterative method is proposed to compute the Hermitian positive definite solution. In the end, an example is used to illustrate the correctness and application of our results.

\section{Introduction}

In this paper we consider the Hermitian positive definite solution of the nonlinear matrix equation

$$
X=Q+\sum_{i=1}^{m} A_{i}\left(B+X^{-1}\right)^{-1} A_{i}^{*}
$$

where $A_{1}, A_{2}, \ldots, A_{m}$ are $n \times n$ complex matrices; $Q$ and $B$ are $n \times n$ Hermitian positive definite matrices. Here, $A_{i}^{*}$ denotes the conjugate transpose of the matrix $A_{i}$. The nonlinear matrix equation (1) plays an important role in linear optimal and robust control. For instance, the solvability of the discrete-time linear quadratic optimal control problem

$$
\begin{array}{ll}
\min _{u_{k}} & \frac{1}{2} \sum_{k=0}^{\infty}\left[\begin{array}{l}
x_{k} \\
u_{k}
\end{array}\right]^{*}\left[\begin{array}{ll}
M_{d} & N_{d} \\
N_{d}^{*} & R_{d}
\end{array}\right]\left[\begin{array}{l}
x_{k} \\
u_{k}
\end{array}\right] \\
\text { s.t. } & E_{d} x_{k+1}=A_{d} x_{k}+B_{d} u_{k}, \quad x_{0}=x^{0},
\end{array}
$$

with $M_{d}^{*}=M_{d}, R_{d}^{*}=R_{d}$ depends on the solvability of (1) in some special cases [1-4]. Due to the important applications in system and control theory, in the past decades, (1) with $m=1$ has been extensively studied, and the research results mainly concentrated on the following:

(a) sufficient conditions and necessary conditions for the existence of an Hermitian solution [5-9];

(b) numerical methods for computing the Hermitian solution $[4,10-13]$;

(c) properties of the Hermitian solution [14, 15];

(d) perturbation analysis for the discrete algebraic Riccati equation [16-18].

(e) connection with symplectic matrix pencil $[9,19,20]$;

(f) connection with stochastic realization and spectral factorization [21-23].

Nonetheless, (1) with $m>1$ has not been studied as far as we know.

In this paper we study the generalized nonlinear matrix equation (1). Firstly, we transform (1) into an equivalent nonlinear matrix equation. By Sherman-Woodbury-Morrison formula [24, Page 50], we have

$$
\left(B+X^{-1}\right)^{-1}=B^{-1}-B^{-1}\left(B^{-1}+X\right)^{-1} B^{-1} ;
$$


then

$$
\begin{aligned}
X & =Q+\sum_{i=1}^{m} A_{i}\left(B+X^{-1}\right)^{-1} A_{i}^{*} \\
& =Q+\sum_{i=1}^{m} A_{i}\left[B^{-1}-B^{-1}\left(B^{-1}+X\right)^{-1} B^{-1}\right] A_{i}^{*} \\
& =Q+\sum_{i=1}^{m} A_{i} B^{-1} A_{i}^{*}-\sum_{i=1}^{m} A_{i} B^{-1}\left(B^{-1}+X\right)^{-1} B^{-1} A_{i}^{*} .
\end{aligned}
$$

Set

$$
M_{i}=A_{i} B^{-1}, \quad N=Q+\sum_{i=1}^{m} A_{i} B^{-1} A_{i}^{*}
$$

then

$$
X=N-\sum_{i=1}^{m} M_{i}\left(B^{-1}+X\right)^{-1} M_{i}^{*}
$$

That is,

$$
X+\sum_{i=1}^{m} M_{i}\left(B^{-1}+X\right)^{-1} M_{i}^{*}=N
$$

Therefore, the nonlinear matrix equation (1) can be equivalently rewritten as (7). So we first investigate the Hermitian positive definite solution of (7) in Section 2 and then derive some new results on the nonlinear matrix equation (1) by using the matrix transformations (5) in Section 3. Finally, we use an example to illustrate the correctness and application of the results of Section 3.

Throughout this paper, we write $B>0(B \geq 0)$ if the matrix $B$ is Hermitian positive definite (semidefinite). If $B-C$ is Hermitian positive definite (semidefinite), then we write $B>C(B \geq C)$. If an Hermitian positive definite matrix $X$ satisfies $B<X \leq C$, we denote by $X \in(B, C]$. We use $\lambda_{1}(B) \geq \lambda_{2}(B) \geq \cdots \geq \lambda_{n}(B)$ to denote all eigenvalues (each repeated as many times as its algebraic multiplicity) of an $n \times n$ Hermitian matrix $B$. The symbol $\|B\|$ denotes the spectral norm of the matrix $B$.

\section{Hermitian Positive Definite Solution of (7)}

In this section, we first give some sufficient conditions and necessary conditions for the existence of an Hermitian positive definite solution of (7), and then we construct an iterative method to compute the Hermitian positive definite solution. We begin with some lemmas.

Lemma 1 (see [25]). If $A \geq B>0$, then $A^{-1} \leq B^{-1}$.

Lemma 2. If (7) has an Hermitian positive definite solution $X$, then

$$
\sum_{i=1}^{m} M_{i}\left(B^{-1}+N\right)^{-1} M_{i}^{*} \leq N
$$

Proof. Since (7) has an Hermitian positive definite solution $X$, that is,

$$
X+\sum_{i=1}^{m} M_{i}\left(B^{-1}+X\right)^{-1} M_{i}^{*}=N
$$

then

$$
X \leq N
$$

And from Lemma 1 it follows that

$$
\left(B^{-1}+X\right)^{-1} \geq\left(B^{-1}+N\right)^{-1}
$$

which leads to

$$
\begin{aligned}
\sum_{i=1}^{m} M_{i} & \left(B^{-1}+N\right)^{-1} M_{i}^{*} \\
& \leq \sum_{i=1}^{m} M_{i}\left(B^{-1}+X\right)^{-1} M_{i}^{*}=N-X<N ;
\end{aligned}
$$

that is,

$$
\sum_{i=1}^{m} M_{i}\left(B^{-1}+N\right)^{-1} M_{i}^{*} \leq N .
$$

Lemma 3. Suppose that the matrices $M_{i}, M_{2}, \ldots, M_{m}$ are nonsingular; if (7) has an Hermitian positive definite solution $X$, then

$$
X \in\left(\frac{1}{m} \sum_{i=1}^{m} M_{i}^{*} N^{-1} M_{i}-B^{-1}, N\right) .
$$

Proof. Since (7) has an Hermitian positive definite solution $X$, that is,

$$
X+\sum_{i=1}^{m} M_{i}\left(B^{-1}+X\right)^{-1} M_{i}^{*}=N
$$

then

$$
X<N, \quad \sum_{i=1}^{m} M_{i}\left(B^{-1}+X\right)^{-1} M_{i}^{*}<N,
$$

which implies

$$
M_{i}\left(B^{-1}+X\right)^{-1} M_{i}^{*}<N, \quad i=1,2, \ldots, m .
$$

By (17) and Lemma 1 and noting that the matrices $M_{i}$, $M_{2}, \ldots, M_{m}$ are nonsingular, then we have

$$
X>M_{i}^{*} N^{-1} M_{i}-B^{-1}, \quad i=1,2, \ldots, m,
$$

which leads to

$$
m X>\sum_{i=1}^{m} M_{i}^{*} N^{-1} M_{i}-m B^{-1}
$$

that is,

$$
X>\frac{1}{m} \sum_{i=1}^{m} M_{i}^{*} N^{-1} M_{i}-B^{-1} .
$$


By (16) and (20), we have

$$
X \in\left(\frac{1}{m} \sum_{i=1}^{m} M_{i}^{*} N^{-1} M_{i}-B^{-1}, N\right) \text {. }
$$

Theorem 4. Let

$$
\begin{aligned}
\Delta_{1}= & {\left[\lambda_{n}(N)-\lambda_{n}\left(B^{-1}\right)\right]^{2}-4 } \\
& \times\left[\sum_{i=1}^{m} \lambda_{1}\left(M_{i} M_{i}^{*}\right)-\lambda_{n}(N) \lambda_{n}\left(B^{-1}\right)\right], \\
\Delta_{2}= & {\left[\lambda_{1}(N)-\lambda_{1}\left(B^{-1}\right)\right]^{2}-4 } \\
& \times\left[\sum_{i=1}^{m} \lambda_{n}\left(M_{i} M_{i}^{*}\right)-\lambda_{1}(N) \lambda_{1}\left(B^{-1}\right)\right] .
\end{aligned}
$$

If

$$
\Delta_{1} \geq 0, \quad \lambda_{1}(N)-\lambda_{1}\left(B^{-1}\right) \geq \lambda_{n}(N)-\lambda_{n}\left(B^{-1}\right)>0,
$$

then (7) has an Hermitian positive definite solution $\widehat{X}$ and $\widehat{X} \epsilon$ $[\alpha I, \beta I]$, where

$$
\begin{aligned}
& \alpha=\frac{\lambda_{n}(N)-\lambda_{n}\left(B^{-1}\right)+\sqrt{\Delta_{1}}}{2}, \\
& \beta=\frac{\lambda_{1}(N)-\lambda_{1}\left(B^{-1}\right)+\sqrt{\Delta_{2}}}{2} .
\end{aligned}
$$

Proof. Since

$$
\begin{aligned}
& \Delta_{1}= {\left[\lambda_{n}(N)-\lambda_{n}\left(B^{-1}\right)\right]^{2}-4 } \\
& \quad \times\left[\sum_{i=1}^{m} \lambda_{1}\left(M_{i} M_{i}^{*}\right)-\lambda_{n}(N) \lambda_{n}\left(B^{-1}\right)\right] \geq 0, \\
& \lambda_{1}(N)-\lambda_{1}\left(B^{-1}\right) \geq \lambda_{n}(N)-\lambda_{n}\left(B^{-1}\right)>0
\end{aligned}
$$

and noting that

$$
\begin{aligned}
& \sum_{i=1}^{m} \lambda_{n}\left(M_{i} M_{i}^{*}\right)-\lambda_{1}(N) \lambda_{1}\left(B^{-1}\right) \\
& \quad \leq \sum_{i=1}^{m} \lambda_{1}\left(M_{i} M_{i}^{*}\right)-\lambda_{n}(N) \lambda_{n}\left(B^{-1}\right),
\end{aligned}
$$

then we have

$$
\begin{aligned}
\Delta_{2}= & {\left[\lambda_{1}(N)-\lambda_{1}\left(B^{-1}\right)\right]^{2}-4 } \\
& \times\left[\sum_{i=1}^{m} \lambda_{n}\left(M_{i} M_{i}^{*}\right)-\lambda_{1}(N) \lambda_{1}\left(B^{-1}\right)\right] \geq 0 .
\end{aligned}
$$

Therefore, the quadratic equation

$$
\begin{aligned}
x^{2}-[ & \left.\lambda_{n}(N)-\lambda_{n}\left(B^{-1}\right)\right] x \\
& +\left[\sum_{i=1}^{m} \lambda_{1}\left(M_{i} M_{i}^{*}\right)-\lambda_{n}(N) \lambda_{n}\left(B^{-1}\right)\right]=0
\end{aligned}
$$

has a positive root $\alpha$, and the quadratic equation

$$
\begin{aligned}
x^{2}- & {\left[\lambda_{1}(N)-\lambda_{1}\left(B^{-1}\right)\right] x } \\
+ & {\left[\sum_{i=1}^{m} \lambda_{n}\left(M_{i} M_{i}^{*}\right)-\lambda_{1}(N) \lambda_{1}\left(B^{-1}\right)\right]=0 }
\end{aligned}
$$

has a positive root $\beta$, where $\alpha$ and $\beta$ are defined by (24). Now we consider the map

$$
\begin{array}{r}
F(X)=N-\sum_{i=1}^{m} M_{i}\left(B^{-1}+X\right)^{-1} M_{i}^{*}, \\
X \in \Omega=[\alpha I, \beta I] .
\end{array}
$$

Obviously, $\Omega$ is a convex, closed, and bounded set and the map $F$ is continuous on $\Omega$. For arbitrary $X \in \Omega$, since $\alpha$ and $\beta$ are positive roots of (28) and (29), respectively, then we have

$$
\begin{aligned}
\lambda_{n}(F(X))= & \lambda_{n}\left[N-M_{1}\left(B^{-1}+X\right)^{-1} M_{1}^{*}\right. \\
& \left.-\cdots-M_{m}\left(B^{-1}+X\right)^{-1} M_{m}^{*}\right] \\
\geq & \lambda_{n}(N)-\lambda_{1}\left(M_{1}\left(B^{-1}+X\right)^{-1} M_{1}^{*}\right) \\
& -\cdots-\lambda_{1}\left(M_{m}\left(B^{-1}+X\right)^{-1} M_{m}^{*}\right) \\
\geq & \lambda_{n}(N)-\frac{\lambda_{1}\left(M_{1} M_{1}^{*}\right)}{\lambda_{n}\left(B^{-1}\right)+\lambda_{n}(X)} \\
& -\cdots-\frac{\lambda_{1}\left(M_{m} M_{m}^{*}\right)}{\lambda_{n}\left(B^{-1}\right)+\lambda_{n}(X)} \\
\geq & \lambda_{n}(N)-\frac{\lambda_{1}\left(M_{1} M_{1}^{*}\right)}{\lambda_{n}\left(B^{-1}\right)+\alpha}-\frac{\lambda_{1}\left(M_{m} M_{m}^{*}\right)}{\lambda_{n}\left(B^{-1}\right)+\alpha} \\
= & \alpha,
\end{aligned}
$$




$$
\begin{aligned}
\lambda_{1}(F(X))= & \lambda_{1}\left[N-M_{1}\left(B^{-1}+X\right)^{-1} M_{1}^{*}\right. \\
& \left.-\cdots-M_{m}\left(B^{-1}+X\right)^{-1} M_{m}^{*}\right] \\
\leq & \lambda_{1}(N)-\lambda_{n}\left(M_{1}\left(B^{-1}+X\right)^{-1} M_{1}^{*}\right) \\
& -\cdots-\lambda_{n}\left(M_{m}\left(B^{-1}+X\right)^{-1} M_{m}^{*}\right) \\
\leq & \lambda_{1}(N)-\frac{\lambda_{n}\left(M_{1} M_{1}^{*}\right)}{\lambda_{1}\left(B^{-1}\right)+\lambda_{1}(X)} \\
& -\cdots-\frac{\lambda_{n}\left(M_{m} M_{m}^{*}\right)}{\lambda_{1}\left(B^{-1}\right)+\lambda_{1}(X)} \\
\leq & \lambda_{1}(N)-\frac{\lambda_{n}\left(M_{1} M_{1}^{*}\right)}{\lambda_{1}\left(B^{-1}\right)+\beta}-\cdots-\frac{\lambda_{n}\left(M_{m} M_{m}^{*}\right)}{\lambda_{1}\left(B^{-1}\right)+\beta} \\
= & \beta ;
\end{aligned}
$$

that is, $F(X) \in \Omega$, which implies that $F(\Omega) \subseteq \Omega$. By Brouwer's fixed point theorem, the map $G$ has a fixed point $\widehat{X} \in \Omega$, which is the positive definite solution of (7). The theorem is proved.

Theorem 5. If

$$
\sum_{i=1}^{m} M_{i}\left(B^{-1}+X\right)^{-1} M_{i}^{*} \leq N-\frac{1}{m} \sum_{i=1}^{m} M_{i} N^{-1} M_{i}^{*}+B^{-1}
$$

for all $X \in\left[(1 / m) \sum_{i=1}^{m} M_{i}^{*} N^{-1} M_{i}-B^{-1}, N\right]$, then (7) has an Hermitian positive definite solution. Furthermore, if

$$
q=m^{2}\left(\sum_{i=1}^{m}\left\|M_{i}\right\|^{2}\right)\left\|\left(\sum_{i=1}^{m} M_{i} N^{-1} M_{i}^{*}\right)^{-1}\right\|^{2}<1,
$$

then (7) has a unique Hermitian positive definite solution.

Proof. Consider the map

$$
\begin{gathered}
F(X)=N-\sum_{i=1}^{m} M_{i}\left(B^{-1}+X\right)^{-1} M_{i}^{*}, \\
X \in W=\left\{X \mid \frac{1}{m} \sum_{i=1}^{m} M_{i}^{*} N^{-1} M_{i}-B^{-1} \leq X \leq N\right\} .
\end{gathered}
$$

Obviously, $W$ is a convex, closed, and bounded set and the map $F$ is continuous on $W$. If

$$
\sum_{i=1}^{m} M_{i}\left(B^{-1}+X\right)^{-1} M_{i}^{*} \leq N-\frac{1}{m} \sum_{i=1}^{m} M_{i} N^{-1} M_{i}^{*}+B^{-1}
$$

for all $X \in W$, then we have

$$
\begin{aligned}
N & \geq N-\sum_{i=1}^{m} M_{i}\left(B^{-1}+X\right)^{-1} M_{i}^{*} \\
& \geq N-N+\frac{1}{m} \sum_{i=1}^{m} M_{i} N^{-1} M_{i}^{*}-B^{-1} \\
& =\frac{1}{m} \sum_{i=1}^{m} M_{i} N^{-1} M_{i}^{*}-B^{-1} ;
\end{aligned}
$$

that is,

$$
\frac{1}{m} \sum_{i=1}^{m} M_{i} N^{-1} M_{i}^{*}-B^{-1} \leq F(X) \leq N .
$$

Hence, $F(W) \subseteq W$. By Brouwer's fixed point theorem, the map $F$ has a fixed point in $W$, which is the Hermitian positive definite solution of (7).

For arbitrary $X, Y \in W$, we have

$$
\begin{aligned}
\| F(X) & -F(Y) \| \\
= & \|\left[N-\sum_{i=1}^{m} M_{i}\left(B^{-1}+X\right)^{-1} M_{i}^{*}\right] \\
& \quad-\left[N-\sum_{i=1}^{m} M_{i}\left(B^{-1}+Y\right)^{-1} M_{i}^{*}\right] \| \\
= & \| \sum_{i=1}^{m} M_{i}\left(B^{-1}+Y\right)^{-1} M_{i}^{*} \\
& \quad-\sum_{i=1}^{m} M_{i}\left(B^{-1}+X\right)^{-1} M_{i}^{*} \| \\
= & \left\|\sum_{i=1}^{m} M_{i}\left[\left(B^{-1}+Y\right)^{-1}-\left(B^{-1}+X\right)^{-1}\right] M_{i}^{*}\right\| \\
\leq & \sum_{i=1}^{m}\left\|M_{i}\left[\left(B^{-1}+Y\right)^{-1}-\left(B^{-1}+X\right)^{-1}\right] M_{i}^{*}\right\| \\
\leq & \left(\sum_{i=1}^{m}\left\|M_{i}\right\|^{2}\right)\left\|\left(B^{-1}+Y\right)^{-1}-\left(B^{-1}+X\right)^{-1}\right\| \\
\leq & \left(\sum_{i=1}^{m}\left\|M_{i}\right\|^{2}\right) \|\left(B^{-1}+X\right)^{-1}\left[\left(B^{-1}+X\right)-\left(B^{-1}+Y\right)\right] \\
\leq & \left.q\left\|M_{i}\right\|^{2}\right) m^{2}\left\|\left(\sum_{i=1}^{m} \| M_{i} M_{i} N^{-1} M_{i}^{*}\right)^{2}\right\| . \\
\times & \times\left(B^{-1}+Y\right)^{-1} \|
\end{aligned}
$$


Since $q<1$, then $F(X)$ is a contraction map in $W$. By Banach's fixed point theorem, we know that the map $F$ has a unique fixed point in $W$, and this shows that (1) has a unique Hermitian positive definite solution in $\left((1 / m) \sum_{i=1}^{m}\right.$ $\left.M_{i}^{*} N^{-1} M_{i}-B^{-1}, N\right)$. Noting Lemma 3, we know that (1) has a unique Hermitian positive definite solution. The theorem is proved.

Nextly, we give an iterative method to compute the Hermitian positive definite solution of (7).

Theorem 6. If there is a real number $\alpha$ satisfying

$$
N-\alpha I \leq \sum_{i=1}^{m} M_{i}\left(B^{-1}+\alpha I\right)^{-1} M_{i}^{*},
$$

then the matrix sequence $\left\{X_{k}\right\}$ converges the Hermitian positive definite solution of (7), where the sequence $\left\{X_{k}\right\}$ is generated by the following iterative method:

$$
\begin{gathered}
X_{0}=\alpha I, \\
X_{k+1}=N-\sum_{i=1}^{m} M_{i}\left(B^{-1}+X_{k}\right)^{-1} M_{i}^{*}, \quad k=0,1,2, \ldots
\end{gathered}
$$

Proof. Consider the iterative method (40). From (39) it follows that

$$
\begin{aligned}
X_{1} & =N-\sum_{i=1}^{m} M_{i}\left(B^{-1}+X_{0}\right)^{-1} M_{i}^{*} \\
& =N-\sum_{i=1}^{m} M_{i}\left(B^{-1}+\alpha I\right)^{-1} M_{i}^{*} \\
& \leq \alpha I \\
& =X_{0} .
\end{aligned}
$$

Since $X_{1} \leq X_{0}$, then $\left(B^{-1}+X_{1}\right)^{-1} \geq\left(B^{-1}+X_{0}\right)^{-1}$ and

$$
\sum_{i=1}^{m} M_{i}\left(B^{-1}+X_{1}\right)^{-1} M_{i}^{*} \geq \sum_{i=1}^{m} M_{i}\left(B^{-1}+X_{0}\right)^{-1} M_{i}^{*}
$$

which implies that

$$
\begin{aligned}
X_{2} & =N-\sum_{i=1}^{m} M_{i}\left(B^{-1}+X_{1}\right)^{-1} M_{i}^{*} \\
& \leq N-\sum_{i=1}^{m} M_{i}\left(B^{-1}+X_{0}\right)^{-1} M_{i}^{*}=X_{1} .
\end{aligned}
$$

Therefore,

$$
X_{2} \leq X_{1} \leq X_{0}
$$

Assume that when $k=l$, we have $X_{l} \leq X_{l-1}$; then

$$
\begin{aligned}
\left(B^{-1}+X_{l}\right)^{-1} & \geq\left(B^{-1}+X_{l-1}\right)^{-1}, \\
\sum_{i=1}^{m} M_{i}\left(B^{-1}+X_{l}\right)^{-1} M_{i}^{*} & \geq \sum_{i=1}^{m} M_{i}\left(B^{-1}+X_{l-1}\right)^{-1} M_{i}^{*},
\end{aligned}
$$

which implies that

$$
\begin{aligned}
X_{l+1} & =N-\sum_{i=1}^{m} M_{i}\left(B^{-1}+X_{l}\right)^{-1} M_{i}^{*} \\
& \leq N-\sum_{i=1}^{m} M_{i}\left(B^{-1}+X_{l-1}\right)^{-1} M_{i}^{*}=X_{l} .
\end{aligned}
$$

By mathematical induction, we obtain that the matrix sequence $\left\{X_{k}\right\}$ is monotone decreasing. Now we begin to use mathematical induction to show that the matrix sequence $\left\{X_{k}\right\}$ is bounded below by some Hermitian positive definite solution of (7).

When $k=0$, by (39) we have

$$
X_{0}-X=\alpha I-\left[N-\sum_{i=1}^{m} M_{i}\left(B^{-1}+X\right)^{-1} M_{i}^{*}\right] \geq 0 .
$$

Assume that when $k=l$, we have $X_{l} \geq X$; then $\left(B^{-1}+X_{l}\right)^{-1} \leq$ $\left(B^{-1}+X\right)^{-1}$, and for $k=l+1$ we have

$$
\begin{aligned}
X_{l+1}-X= & {\left[N-\sum_{i=1}^{m} M_{i}\left(B^{-1}+X_{l}\right)^{-1} M_{i}^{*}\right] } \\
& -\left[N-\sum_{i=1}^{m} M_{i}\left(B^{-1}+X\right)^{-1} M_{i}^{*}\right] \\
= & \sum_{i=1}^{m} M_{i}\left(B^{-1}+X\right)^{-1} M_{i}^{*} \\
& -\sum_{i=1}^{m} M_{i}\left(B^{-1}+X_{l}\right)^{-1} M_{i}^{*} \\
= & \sum_{i=1}^{m} M_{i}\left[\left(B^{-1}+X\right)^{-1}-\left(B^{-1}+X_{l}\right)^{-1}\right] M_{i}^{*} \\
\geq & 0 .
\end{aligned}
$$

Therefore, the matrix sequence $\left\{X_{k}\right\}$ is a monotonic decreasing sequence and bounded below by some Hermitian positive definite solution $X$, and then it converges to an Hermitian positive definite solution $X$ of (1). The theorem is proved.

\section{Hermitian Positive Definite Solution of (1)}

By the matrix transformations (5), Lemmas 2 and 3, and Theorems 4-6, we have the following theorems.

Theorem 7. If (1) has an Hermitian positive definite solution $X$, then

$$
\begin{gathered}
\sum_{i=1}^{m} A_{i} B^{-1}\left(B^{-1}+Q+\sum_{i=1}^{m} A_{i} B^{-1} A_{i}^{*}\right)^{-1} B^{-1} A_{i}^{*} \\
<Q+\sum_{i=1}^{m} A_{i} B^{-1} A_{i}^{*} .
\end{gathered}
$$


Theorem 8. Suppose that the matrices $A_{1}, A_{2}, \ldots, A_{m}$ are nonsingular; if (1) has an Hermitian positive definite solution $X$, then

$$
\begin{gathered}
X \in\left(\frac{1}{m} \sum_{i=1}^{m} B^{-1} A_{i}^{*}\left(Q+\sum_{i=1}^{m} A_{i} B^{-1} A_{i}^{*}\right)^{-1} A_{i} B^{-1}-B^{-1},\right. \\
\left.Q+\sum_{i=1}^{m} A_{i} B^{-1} A_{i}^{*}\right) .
\end{gathered}
$$

Remark 9. The upper bounds of Hermitian solution for the discrete algebraic Riccati equation have been extensively studied in the past three decades (see [14] and references therein). Compared with previous works, one gives a new upper bound of Hermitian solution but also gives a lower bound in Theorem 8 .

Theorem 10. Let

$$
\begin{aligned}
\Delta_{1}^{\prime}= & {\left[\lambda_{n}\left(\mathrm{Q}+\sum_{i=1}^{m} A_{i} B^{-1} A_{i}^{*}\right)-\lambda_{n}\left(B^{-1}\right)\right]^{2} } \\
& -4\left[\sum_{i=1}^{m} \lambda_{1}\left(A_{i} B^{-2} A_{i}^{*}\right)-\lambda_{n}\left(Q+\sum_{i=1}^{m} A_{i} B^{-1} A_{i}^{*}\right)\right. \\
& \left.\times \lambda_{n}\left(B^{-1}\right)\right] ; \\
\Delta_{2}^{\prime}= & {\left[\lambda_{1}\left(Q+\sum_{i=1}^{m} A_{i} B^{-1} A_{i}^{*}\right)-\lambda_{1}\left(B^{-1}\right)\right]^{2} } \\
& -4\left[\sum_{i=1}^{m} \lambda_{n}\left(A_{i} B^{-2} A_{i}^{*}\right)\right. \\
& \left.-\lambda_{1}\left(Q+\sum_{i=1}^{m} A_{i} B^{-1} A_{i}^{*}\right) \lambda_{n}\left(B^{-1}\right)\right] .
\end{aligned}
$$

If

$$
\begin{gathered}
\Delta_{1}^{\prime} \geq 0, \\
\lambda_{1}\left(Q+\sum_{i=1}^{m} A_{i} B^{-1} A_{i}^{*}\right)-\lambda_{1}\left(B^{-1}\right) \\
\geq \lambda_{n}\left(Q+\sum_{i=1}^{m} A_{i} B^{-1} A_{i}^{*}\right)-\lambda_{n}\left(B^{-1}\right)>0,
\end{gathered}
$$

then (1) has an Hermitian positive definite solution $X$, and $X \in$ $\left[\alpha^{\prime} I, \beta^{\prime} I\right]$, where

$$
\begin{aligned}
& \alpha^{\prime}=\frac{\lambda_{n}\left(Q+\sum_{i=1}^{m} A_{i} B^{-1} A_{i}^{*}\right)-\lambda_{n}\left(B^{-1}\right)+\sqrt{\Delta_{1}^{\prime}}}{2}, \\
& \beta^{\prime}=\frac{\lambda_{1}\left(Q+\sum_{i=1}^{m} A_{i} B^{-1} A_{i}^{*}\right)-\lambda_{1}\left(B^{-1}\right)+\sqrt{\Delta_{2}^{\prime}}}{2} .
\end{aligned}
$$

Theorem 11. If

$$
\begin{aligned}
\sum_{i=1}^{m} A_{i} & B^{-1}\left(B^{-1}+X\right)^{-1} B^{-1} A_{i}^{*} \\
\leq & Q+\sum_{i=1}^{m} A_{i} B^{-1} A_{i}^{*} \\
& \quad-\frac{1}{m} \sum_{i=1}^{m} A_{i} B^{-1}\left(Q+\sum_{i=1}^{m} A_{i} B^{-1} A_{i}^{*}\right)^{-1} B^{-1} A_{i}^{*}+B^{-1}
\end{aligned}
$$

for all $X \in\left[(1 / m) \sum_{i=1}^{m} B^{-1} A_{i}^{*}\left(Q+\sum_{i=1}^{m} A_{i} B^{-1} A_{i}^{*}\right)^{-1} B^{-1} A_{i}-\right.$ $\left.B^{-1}, Q+\sum_{i=1}^{m} A_{i} B^{-1} A_{i}^{*}\right]$, then (1) has an Hermitian positive definite solution. Furthermore, if

$$
\begin{aligned}
q= & m^{2}\left(\sum_{i=1}^{m}\left\|A_{i} B^{-1}\right\|^{2}\right) \\
& \times\left\|\left(\sum_{i=1}^{m} A_{i} B^{-1}\left(Q+\sum_{i=1}^{m} A_{i} B^{-1} A_{i}^{*}\right)^{-1} B^{-1} A_{i}^{*}\right)^{-1}\right\|^{2} \\
& <1
\end{aligned}
$$

then (1) has a unique Hermitian positive definite solution.

Theorem 12. If there is a real number $\alpha^{\prime}$ satisfying

$$
\begin{aligned}
& Q+\sum_{i=1}^{m} A_{i} B^{-1} A_{i}^{*}-\alpha^{\prime} I \\
& \quad \leq \sum_{i=1}^{m} A_{i} B^{-1}\left(B^{-1}+\alpha^{\prime} I\right)^{-1} B^{-1} A_{i}^{*}
\end{aligned}
$$

then the matrix sequence $\left\{X_{k}\right\}$ converges to the Hermitian positive definite solution of (1), where the matrix sequence $\left\{X_{k}\right\}$ is generated by the following iterative method:

$$
\begin{gathered}
X_{0}=\alpha^{\prime} I, \\
X_{k+1}=Q+\sum_{i=1}^{m} A_{i} B^{-1} A_{i}^{*}-\sum_{i=1}^{m} A_{i} B^{-1}\left(B^{-1}+X_{k}\right)^{-1} B^{-1} A_{i}^{*}, \\
k=0,1,2, \ldots
\end{gathered}
$$

\section{Numerical Experiments}

In this section, we give an example to demonstrate the correctness and application of our results.

Example 1. Consider the following discrete-time LQR optimal control problem:

$$
\begin{array}{r}
\min _{u[0, \cdot] N \rightarrow \infty} \lim _{N \rightarrow} J_{N} \text { w.r.t } x(k+1)=A x(k)+u(k), \\
x(0)=x_{0}, \quad x(\cdot), u(\cdot) \subset R^{4}
\end{array}
$$


with the additional constraint that $\lim _{N \rightarrow \infty} x(N)=0$, where

$$
J_{N}=\frac{1}{2} \sum_{k=0}^{N-1}\left\{x^{T}(k) Q x(k)+u^{T}(k) u(k)\right\},
$$

$x_{0}=[0.45,0.72,-0.67,-0.16]^{T}$, and both $A$ and $Q$ are as follows:

$$
\begin{aligned}
& A=\left(\begin{array}{cccc}
8 & 2 & -3 & 4 \\
-8 & 2 & 3 & 4 \\
2 & 3 & 4 & 5 \\
2 & 1 & -1 & 7
\end{array}\right), \\
& Q=\left(\begin{array}{cccc}
292 & -18 & -48 & 60 \\
-18 & 94 & -36 & 54 \\
-48 & -36 & 186 & 22 \\
60 & 54 & 22 & 232
\end{array}\right)
\end{aligned}
$$

The optimal control sequence that minimizes the performance index is $u(k)=-K x(k)$, where $K=(I+X)^{-1} X A$ and $X=X^{T}>0$ satisfies the discrete algebraic Riccati equation

$$
\begin{aligned}
X & =Q+A\left[X-X(I+X)^{-1} X\right] A^{T} \\
& =Q+A\left(I+X^{-1}\right)^{-1} A^{T} .
\end{aligned}
$$

It is easy to verify that the nonlinear matrix equation (62) satisfies the conditions of Theorem 10; hence, (62) has an Hermitian positive definite solution $X$. Now we use iterative method (58) to compute the Hermitian positive definite solution of (62). Let $\alpha=58.6$, and after 6 iterations, we get the positive definite solution

$$
X \approx X_{6}=\left(\begin{array}{cccc}
384.74 & -70.83 & -18.07 & 108.90 \\
-70.83 & 186.66 & -14.05 & 64.88 \\
-18.07 & -14.05 & 239.83 & 59.93 \\
108.90 & 64.88 & 59.93 & 286.78
\end{array}\right)
$$

and its residual error $R\left(X_{6}\right)=\left\|X_{6}-A\left(I+X_{6}^{-1}\right)^{-1} A^{T}-Q\right\| \approx$ $3.28 \times 10^{-15}$. Of course it is easy to verify that

$$
X \approx X_{6} \in\left(A^{*}\left(Q+A A^{*}\right)^{-1} A, Q+A A^{*}\right),
$$

which confirms the correctness of Lemma 3.

From

$$
\begin{aligned}
u(k) & =-K x(k)=-(I+X)^{-1} X A x(k) \\
& \approx-\left(I+X_{6}\right)^{-1} X_{6} A x(k),
\end{aligned}
$$

we have

$$
x(k+1)=A x(k)+u(k) \approx\left[A-\left(I+X_{6}\right)^{-1} X_{6} A\right] x(k) ;
$$

hence, $x(k) \approx\left[A-\left(I+X_{6}\right)^{-1} X_{6} A\right]^{k} x_{0}$. Then taking $N=36$ we have

$$
\begin{aligned}
J_{N} & \approx \frac{1}{2} \sum_{k=0}^{N-1} x^{T}(k)\left[Q+A^{T} X_{6}\left(I+X_{6}\right)^{-2} X_{6} A\right] x(k) \\
& =298.4449 .
\end{aligned}
$$

\section{Conclusion}

In this paper, we consider the Hermitian positive definite solution of the nonlinear matrix equation $X=Q+\sum_{i=1}^{m} A_{i}(B+$ $\left.X^{-1}\right)^{-1} A_{i}^{*}$, which plays an important role in linear optimal and robust control. By making use of Sherman-WoodburyMorrison formula, we transform this equation into an equivalent nonlinear matrix equation. So we can investigate this nonlinear matrix equation by the aid of the equivalent nonlinear matrix equation. Some new sufficient conditions and necessary conditions for the existence of an Hermitian positive definite solution are derived. An iterative method is proposed to compute its Hermitian positive definite solution. In the end, an example is used to illustrate the correctness and application of our results.

\section{Conflict of Interests}

The authors declare that there is no conflict of interests regarding the publication of this paper.

\section{Acknowledgments}

The work was supported by the National Natural Science Foundation of China (nos. 11101100, 11301107, and 11261014) and the Natural Science Foundation of Guangxi Province (nos. 2012GXNSFBA053006 and 2013GXNSFBA019009).

\section{References}

[1] D. J. Bender and A. J. Laub, "The linear-quadratic optimal regulator for descriptor systems," IEEE Transactions on Automatic Control, vol. 32, no. 8, pp. 672-688, 1987.

[2] P. Lancaster and L. Rodman, Algebraic Riccati Equations, Oxford University Press, Oxford, UK, 1995.

[3] B. D. Anderson and J. B. Moore, Linear Optimal Control, Prentice Hall, Englewood Cliffs, NJ, USA, 1971.

[4] T. Pappas, A. J. Laub, and N. R. Sandell, "On the numerical solution of the discrete-time algebraic Riccati equation," IEEE Transactions on Automatic Control, vol. 25, no. 4, pp. 631-641, 1980.

[5] D. J. Clements and H. K. Wimmer, "Existence and uniqueness of unmixed solutions of the discrete-time algebraic Riccati equation," Systems \& Control Letters, vol. 50, no. 5, pp. 343-346, 2003.

[6] J. C. Engwerda, A. C. Ran, and A. L. Rijkeboer, "Necessary and sufficient conditions for the existence of a positive definite solution of the matrix equation $X+A^{*} X^{-1} A=Q$," Linear Algebra and Its Applications, vol. 186, pp. 255-275, 1993.

[7] A. Ferrante and B. C. Levy, "Hermitian solutions of the equation $X=Q+N X^{-1} N^{*}$," Linear Algebra and its Applications, vol. 247, pp. 359-373, 1996.

[8] G. Freiling, V. Mehrmann, and H. Xu, "Existence, uniqueness, and parametrization of Lagrangian invariant subspaces," SIAM Journal on Matrix Analysis and Applications, vol. 23, no. 4, pp. 1045-1069, 2002.

[9] H. K. Wimmer, "Normal forms of symplectic pencils and the discrete-time algebraic Riccati equation," Linear Algebra and Its Applications, vol. 147, pp. 411-440, 1991. 
[10] P. Benner, Contributions to the Numerical Solution of Algebraic Riccati Equations and Related Eigenvalue Problems, Logos, Berlin, Germany, 1997.

[11] P. Van Dooren, "A generalized eigenvalue approach for solving Riccati equations," Society for Industrial and Applied Mathematics, vol. 2, no. 2, pp. 121-135, 1981.

[12] L. Z. Lu and W. W. Lin, "An iterative algorithm for the solution of the discrete-time algebraic Riccati equation," Linear Algebra and Its Applications, vol. 188-189, pp. 465-488, 1993.

[13] A. J. Laub, "A Schur method for solving algebraic Riccati equations," IEEE Transactions on Automatic Control, vol. 24, no. 6, pp. 913-921, 1979.

[14] R. Davies, P. Shi, and R. Wiltshire, "Upper solution bounds of the continuous and discrete coupled algebraic Riccati equations," Automatica, vol. 44, no. 4, pp. 1088-1096, 2008.

[15] I. G. Ivanov, "Properties of Stein (Lyapunov) iterations for solving a general Riccati equation," Nonlinear Analysis: Theory, Methods \& Applications, vol. 67, no. 4, pp. 1155-1166, 2007.

[16] M. M. Konstantinov, P. H. Petkov, and D. W. Gu, "Improved perturbation bounds for general quadratic matrix equations," Numerical Functional Analysis and Optimization, vol. 20, no. 78, pp. 717-736, 1999.

[17] J. G. Sun, "Sensitivity analysis of the discrete-time algebraic Riccati equation," in Proceedings of the 6th Conference of the International Linear Algebra Society, vol. 275/276 of Linear Algebra and its Applications, pp. 595-615.

[18] L. Zhou, Y. Lin, Y. Wei, and S. Qiao, "Perturbation analysis and condition numbers of symmetric algebraic Riccati equations," Automatica, vol. 45, no. 4, pp. 1005-1011, 2009.

[19] A. Ferrante and B. C. Levy, "Canonical form of symplectic matrix pencils," Linear Algebra and its Applications, vol. 274, pp. 259-300, 1998.

[20] A. Ferrante and L. Ntogramatzidis, "A unified approach to finite-horizon generalized LQ optimal control problems for discrete-time systems," Linear Algebra and its Applications, vol. 425, no. 2-3, pp. 242-260, 2007.

[21] A. Ferrante, "A parameterization of minimal stochastic realizations," IEEE Transactions on Automatic Control, vol. 39, no. 10, pp. 2122-2126, 1994.

[22] A. Lindquist and G. Picci, "Realization theory for multivariate stationary Gaussian processes," SIAM Journal on Control and Optimization, vol. 23, no. 6, pp. 809-857, 1985.

[23] A. Lindquist and G. Picci, "A geometric approach to modelling and estimation of linear stochastic systems," Journal of Mathematical Systems, Estimation, and Control, vol. 1, no. 3, pp. 241333, 1991.

[24] G. H. Golub and C. F. Van Loan, Matrix Computations, Johns Hopkins University, London, UK, 3rd edition, 1996.

[25] X. Zhan, Matrix Inequalities, Springer, Berlin, Germany, 2002. 


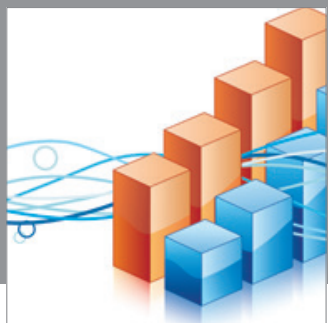

Advances in

Operations Research

mansans

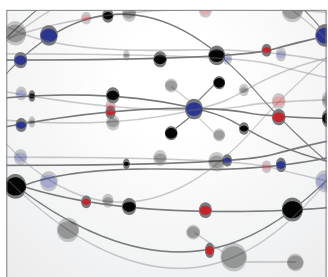

The Scientific World Journal
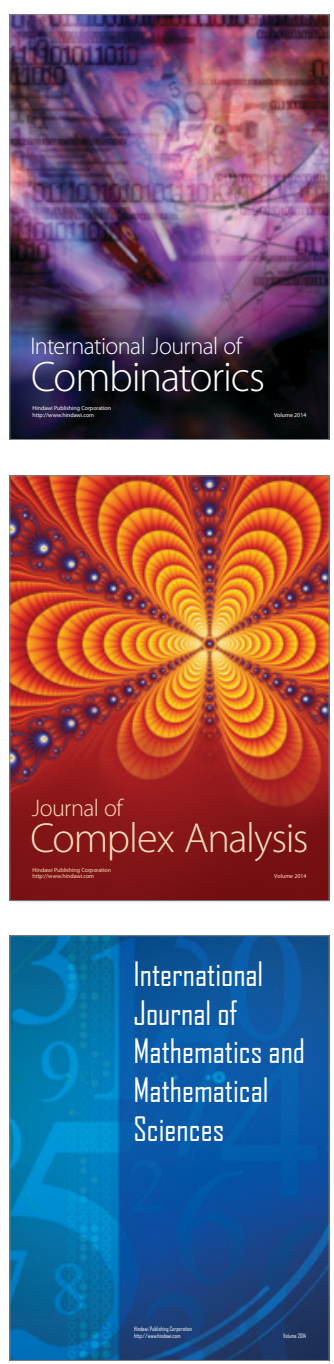
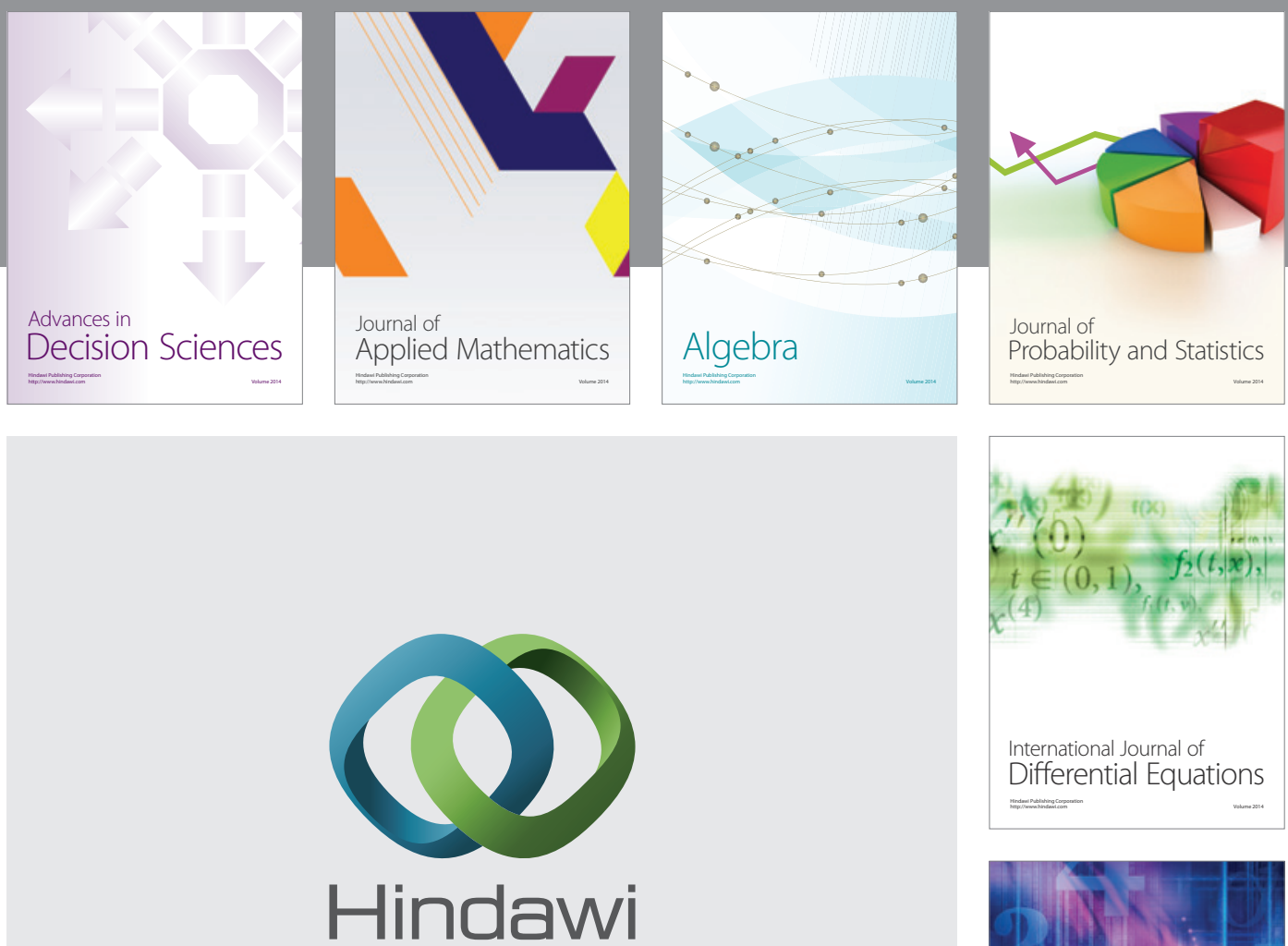

Submit your manuscripts at http://www.hindawi.com
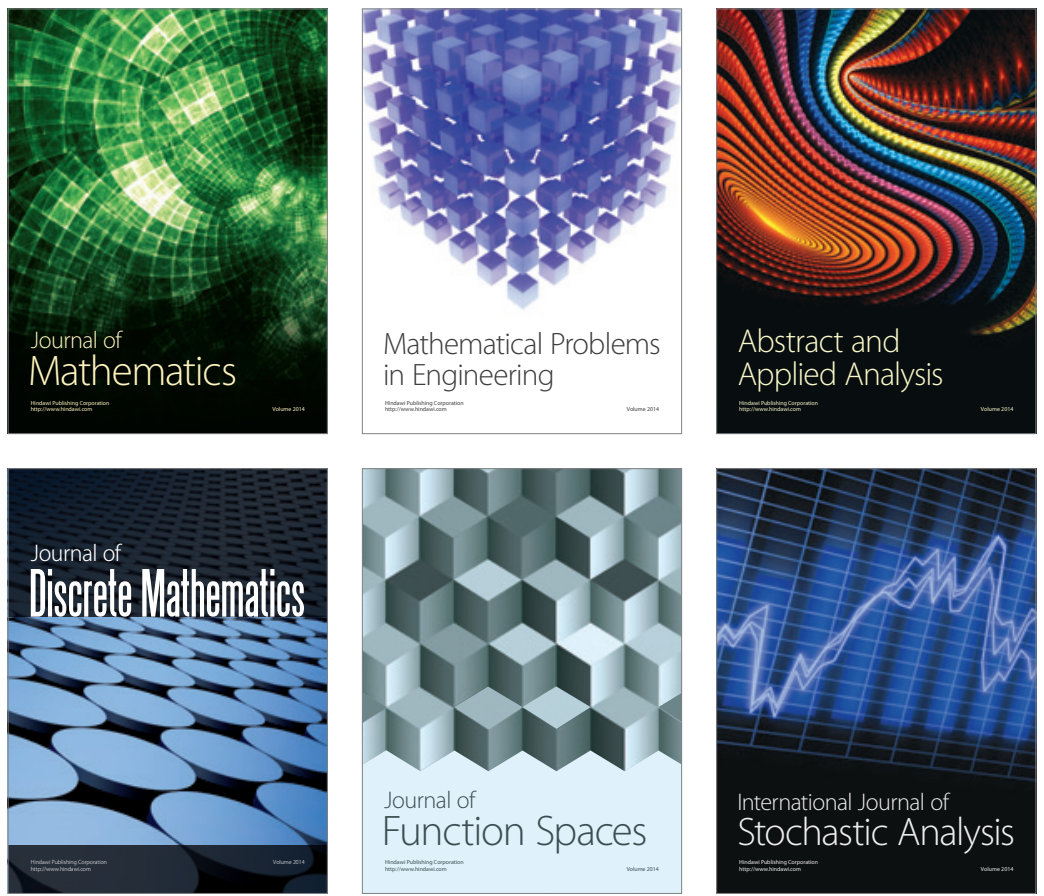

Journal of

Function Spaces

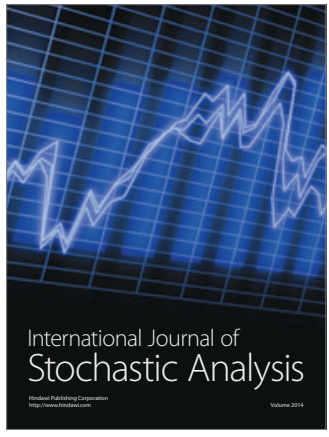

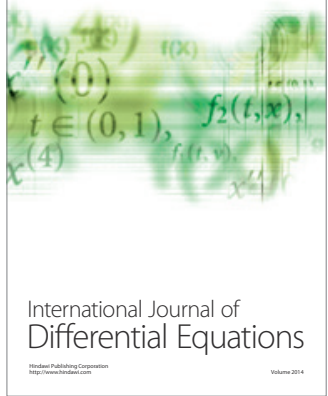
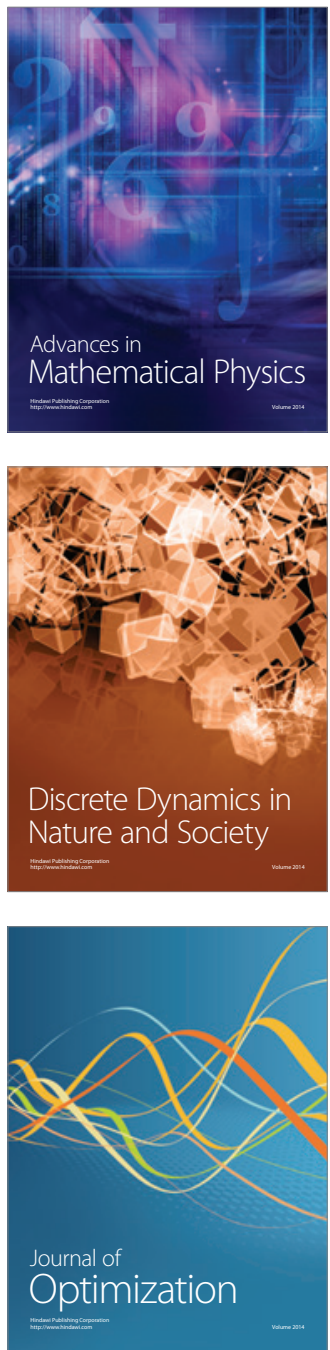